\title{
PRODUCT DESIGN EDUCATION IN MECHANICAL AND MANAGEMENT ENGINEERING MASTER'S DEGREE PROGRAMMES. ANALOGIES AND DIFFERENCES
}

\author{
Sergio RIZZUTI and Luigi DE NAPOLI \\ University of Calabria - DIMEG, Ponte Pietro Bucci 46/C, 87030 Rende (CS), Italy
}

\begin{abstract}
Mechanical and Management Engineering are some of the stakeholders involved in product development, with different competencies. They must share the responsibility for best solution identification to accordingly answer the customer needs and to authorize the production of products that can have success with a positive economic return.

Higher education curricula should prepare such professionals, and this is the reason because courses of product design are proposed to such classes. Even the programmes of the corresponding courses are necessarily different the part related to concept generation is similar.

The work aims is to identify analogies and differences between the classes of Mechanical Engineering and Management Engineering, both in the way of leading the generation of concepts and in how the concepts generated have eventually reached the goals of innovation.

BrainWriting is a method that can allow students to be productive in concept generation and is based on the two steps of sketching and gallery. This ability must be conjointly employed with the functional study of the problem to be solved. These aspects must be learned by students to avoid fixation, the inconvenience to repeat indefinitely only what is already known.

The design alternatives generated by teams of students will be assessed by teachers and experts in the field, following the method proposed by Shah. After such classification, further comparison between the two classes will be done to identify the aspects of the cultural imprinting of such future professionals.
\end{abstract}

Keywords: Product design education, creativity and innovation, BrainWriting, comparison between different classes, creativity assessment

\section{INTRODUCTION}

Courses related to product design are provided in industrial engineering academic degree programmes, worldwide, following the most varied design methods [1-3]. In each of such courses, a fundamental step is when the concept generation phase is introduced, the phase in which the designer's request for the creative effort is at its maximum.

The methods used to generate the concepts are several [1] and attain to the sensibility of teachers and/or the audience to the people involved. Also, the teaching methods (PBL or direct lectures) influence the right choice.

In this work, we will describe how the concept generation phase is carried out during a group work of different classes of students and the results will be accordingly evaluated.

In this experience, a collection of methods, named BrainWriting [4] according to procedures refined over the years, are used in two classes of students attending the Management and Mechanical Engineering master's degree.

The first class concerns students with little experience and not very familiar with the problems of designing an industrial product; these are postgraduate students in Management Engineering attending the second semester of a master's degree. The second class concerns more experienced students, belonging to a class of the fourth semester of the master's degree in Mechanical Engineering, students very close to the degree, who have already faced various engineering problems.

The theme proposed this year is unique for both classes and the same protocol is followed as a variant of the BrainWriting method, performed in several sessions [5]. 
The preparation for the concept generation sessions is based on the customer needs that the product should have to satisfy and the functional analysis of the correspondent industrial product typology.

The generated concepts are assessed by experts from both the academic and the industrial world, using the metrics proposed by Shah et al $[6,7]$.

During the generation of concepts, emphasis will be placed on how the two classes deal with preparation, incubation, and illumination phases.

The aim of the work is to identify analogies and differences both in the way of leading the generation of concepts and in how the concepts generated have eventually reached the goals of innovation.

This represents a further step towards the definition of the most appropriate procedures to become more productive in the generation of innovative concepts, depending on or regardless of the students' level of awareness of engineering problems and their maturity.

\section{CONTEXTS}

Over the years, it has been recognized certain importance in courses of Product Design at the Department of Mechanical, Energy and Management Engineering (DIMEG) of the University of Calabria (Italy). In both master's degree courses in Mechanical and Management Engineering, such kinds of courses were established since the year 2000. Obviously, the emphasis given to these courses is different, due to the different curricula followed by students. Also, the course name corresponds to such a difference. As reported in Table 1, the topics treated have a certain difference, because the finalities are different.

Table 1. Programmes and finality of the course

\begin{tabular}{|c|c|c|}
\hline \multirow{2}{*}{ phases } & Mechanical Engineering class & \multirow{2}{*}{$\begin{array}{l}\text { Management Engineering class } \\
\text { Sustainable Product Design }\end{array}$} \\
\hline & Product Design and Development & \\
\hline $\begin{array}{l}\text { Problem } \\
\text { clarification }\end{array}$ & $\begin{array}{l}\text { The nature of design and the need for } \\
\text { systematic approaches. } \\
\text { The steps of product design and development } \\
\text { process. The product life cycle. } \\
\text { Identification of market opportunity. } \\
\text { Identification of customer needs; product } \\
\text { planning; the requirement list. }\end{array}$ & $\begin{array}{l}\text { Identifying customer needs and product } \\
\text { specifications. }\end{array}$ \\
\hline $\begin{array}{l}\text { Concept } \\
\text { design }\end{array}$ & $\begin{array}{l}\text { The functional approach to design; the } \\
\text { functional net: clustered graph and function } \\
\text { tree. } \\
\text { Concept generation: search internally, } \\
\text { individually and in the group; externally } \\
\text { activities. Design creativity. } \\
\text { Concept selection. Matrix of relative and } \\
\text { absolute importance: screening and scoring. }\end{array}$ & $\begin{array}{l}\text { Functional analysis of a product. } \\
\text { Reverse engineering of industrial products by } \\
\text { means of functional decomposition. } \\
\text { Concept generation and selection. }\end{array}$ \\
\hline $\begin{array}{l}\text { Embodiment } \\
\text { design }\end{array}$ & $\begin{array}{l}\text { Product architecture and product structure. } \\
\text { Introduction to DSM (Design Structure } \\
\text { Matrix) } \\
\text { Axiomatic Design and design matrix. } \\
\text { DfM (Design for Manufacturing) and DfA } \\
\text { (Design for Assembly). } \\
\text { DfE (Design for Environment). } \\
\text { Robust Design (Taguchi method) and } \\
\text { ANOVA. } \\
\text { Quality Function Deployment and the House } \\
\text { of Quality. } \\
\text { Customer satisfaction (Kano model). }\end{array}$ & $\begin{array}{l}\text { Product architecture and embodiment design. } \\
\text { Design for Environment. } \\
\text { Environmental problems and strategies for } \\
\text { sustainability. } \\
\text { Product life thinking. } \\
\text { Product Lifecycle Management (PLM). } \\
\text { QFD - House of Quality. } \\
\text { Customer satisfaction (Kano model). } \\
\text { Design for X: Design for Assembly, Design } \\
\text { for Manufacturing, Design for Disassembly, } \\
\text { etc.. }\end{array}$ \\
\hline Detail design & $\begin{array}{l}\text { Detail design and the geometric quality of the } \\
\text { industrial products. }\end{array}$ & $\begin{array}{l}\text { Design of an industrial product, from customer } \\
\text { needs to the embodiment design and an early } \\
\text { LCA. }\end{array}$ \\
\hline
\end{tabular}

Mechanical Engineering students must collect and synthesize all the knowledge acquired along with their curriculum, solving a problem arriving to present a project in which the assembly and the detail design are presented professionally, with the check of all the rules and norms. The final report should 
contain a discussion of all the steps followed and Robust Design considerations, derived by computer simulation of main physical aspects of the device.

Management Engineering should stress mainly the relationship between the final product and the initial customer needs. Further attempts will be dedicated to the Kano model for customer satisfaction, Quality Function Deployment, and Design for Environment. Students' final task is the writing of a report in which all the steps followed are discussed, focusing on sustainability, concept design, methods for supporting decisions among alternative solutions. Both courses are organized subdividing the audience into teams ranging from 2 to 4 people. The paradigm of Project-Based Learning has proven to be valuable for the educational scope of such teachings.

Obviously, being the final task of the courses considerably different, a comparison will be made on the initial part of them. The part of conceptual design is performed in the same way, by the 5 steps described in Ulrich-Eppinger [2]. The strict phase of concept generation is performed by BrainWriting [1, 5, 8]. At the basis of this method, there is the sketching of ideas on a sheet of paper, on which each member of the team, after a first elaboration of his/her own ideas, is free to add a positive contribution to the work done by the other components of the team.

Freehand sketching [9] is the main way in which everyone freely describes one or more ideas. Such kind of representation is well understood by students of both courses because they mandatory followed courses of Engineering Drawing during their respective bachelor's degrees. In any way, the two initial weeks of these courses present a laboratory for improving this ability on sketching.

A further novelty of this year will consist of the employment of SketchBook® [10] during a virtual BrainWriting session. This is due to the health emergency that now involves Italy, because of COVID19 infection, and obliged to e-learning. To allow interactivity among students the Microsoft Teams ${ }^{\circledR}$ ambient was used.

\section{EXPERIMENT}

The experiment is generally performed during the first phases of the conceptual design of an industrial product in which, once decided on the problem to be solved and planned the required functionalities, a design team generates the first conceptual solutions. Both courses are held in the second semester of this academic year 2019-2020 since the half of March. The experiment this year has been performed in both classes in the fourth and fifth weeks of the semester schedule.

Being different the number of students of the two classes involved: 8 students in Mechanical Engineering and 20 students in Management Engineering a certain caution will be required in the phase of statistical elaboration of data. Students of the Mechanical Engineering class self-organized into 2 groups of four and students of the Management Engineering class in 4 groups of three or 2 groups of four.

In the first three weeks, in both classes, a set of activities were performed, led by the two teachers. In the first week, the needs hidden into the product to be designed and related to the problem to be solved are exposed and examined. The theme on which students decided to be engaged has been "allow to frequent a gym for fitness in time of COVID-19", that could materialize in a "mask". An external survey to understand better the real needs around this problem was autonomously organized by students. In the following week, the problem was analysed by functional analysis.

After this preliminary session, students had a week to enlarge the context of their knowledge around the problem with external research. This time was also necessary to incubate their own ideas. After that, a set of channels were organized in the Microsoft Teams ${ }^{\circledR}$ ambient in which each team, in their own channel, carried out the concept generation session.

For the concept generation, a BrainWriting (BW) method, refined over the years [5], has been used. It is a variant of the 6-3-5 method, followed by a gallery method.

In the present edition of the BW session, the following structure was adopted: the number of participants was three or four, for each team; each team member had 15 minutes to design the initial concepts and 10 minutes for each successive improvement phase, where each participant marked improvements to the drawing in front of him; the total duration of a session was fixed around 45 minutes; the timing has been checked by teachers and the participants had the denial of discussing each other. This year the first sketches were drawn on paper that was transferred as an image, by means of a JPEG file, to the other team components. The improvements were added by Sketchbook ${ }^{\circledR}$ on a different layer identified by a different colour, one associated to a different team member. The BrainWriting session ended with the 
gallery of the concepts generated, within each group, to refine, classify, structure, and prepare all the material produced, for the subsequent phase of the concept selection.

The main difference between the two classes was the approach to concept generation. In the Mechanical Engineering class, the main function was broken down using the classic verb/noun paradigm, with any potential attributes, arriving at a level of decomposition that allowed students to engage in the association of several ideas to each of them. Alongside the functional analysis session, the teacher paid attention to do not employ himself and not allow students to use terms that explicitly remind them to already existing solutions. In the Management Engineering class, the problem was investigated as a whole, taking in mind all the aspects that emerged during the reverse engineering session. This had consequently a different number of ideas generated during BrainWriting, which cannot be related to the different curriculum of the students, but instead to the different approaches to concept generation.

\section{METRICS}

The main task of the paper is to find if any, analogies and/or differences between the two classes of students that are involved in the process of product design, that have a different cultural background. The phase of concept generation was proposed and performed in a slightly different way, as reported above, so it is important to identify the right set of parameters that must be extracted and assessed from the elaborations, and that can allow to differentiate the two classes. A certain discrepancy could also be present inside the two classes considering the different number of students, which could have introduced a further noise in the investigation.

The parameters must be quantitative and qualitative, and they must be identified as a priori.

Because creativity is a complex matter, with a great variety of levels, it continues to have an elusive nature. Many methods for assessing creativity have been suggested and investigated [11]. The choice of applying a method is not trivial and this depends very much on the context in which the creative process applies [12]. The most used methods in the industrial context are the Consensual Assessment Technique (CAT) [13] and the Torrance Test of Creative Thinking (TTCT) [14]. Besides these, the method proposed by Shah at al. [6,7] is really promising for the application in engineering design, considering that the conceived items must satisfy a set of specifications.

To be able to differentiate among design proposals, it is necessary to establish some parameters that can be related to the main product characteristics. Four dimensions are suggested by Shah et al.: quantity, quality, novelty, and variety. These parameters will be evaluated for each design solution that each team will have elaborated after the gallery session, in which each team will have ordered and classified all alternatives to the functionalities on which the BrainWriting session has been focused. Quantity is just the ideas count. Quality is almost difficult to identify without a prototype (physical or digital) and it will not be processed, considering the drafts related to the conceptual phase, where cannot be identified quantitative information [7].

Novelty and variety require the intervention of evaluators. Three will be the evaluators: the two teachers, and one expert that comes from the industrial world. He is a designer of electromechanical devices operating in a wide variety of contexts.

\section{RESULTS AND DISCUSSION}

The experiment was carried out in April 2020. Students of both classes were surprised the activity was really engaging and that in a short lapse of time they were able to produce something interesting.

The different approaches followed in the idea generation within the two classes induced the generation of a slightly different number of devices. Just to say, the Management Engineering teams generated several items exactly coincident to the number of students, because they conceived devices. Mechanical Engineering, after the conceptualization of several solutions for each functionality and after the classification of those in a table, generated many solutions as a rational combination of components. The two Mechanical teams produced quite the same number of devices ( 8 and 9$)$. One team added further other 3 devices after the screening when the students merged some basic ideas in new items. Therefore, in the end, the number of devices that globally was invented by both classes was the same: 20 items per class. Therefore, quantity cannot be applied as a parameter able to differentiate between the classes. And quality will not be processed.

Novelty is here assessed identifying the key attributes of the device, classifying all different ways in which these attributes are satisfied and privileging the ideas with the lower counting. Initially, novelty is associated to each item conceived. Then these assesses are grouped per class and the distributions are 
compared. The device has been identified by 4 macro functions; F1- Filter in input; F2- Filter in output; F3- Adhere to face; F4- Fix to head. A weight has been associated with each of them $\left(f_{l}=0.3 ; f_{2}=0.3\right.$; $f_{3}=0.25 ; f_{4}=0.15$ ).

Novelty for each single $j$-th item is defined as $\quad M_{N_{j}}=\sum_{i=1}^{4} f_{i} \cdot S_{N_{i j}}$

Where $S_{N_{i j}}$ is the novelty score of $j$-th item for $i$-th functionality,

with $j=1, T ; T=20$ items; $S_{N_{i j}}=\frac{T-C_{i j}}{T}$, where $C_{i j}$ is the count of the current item for that function. The novelty for each class can be shown as a box in Figure 1, which allows comparison. In any case, an average novelty value may be evaluated for each class (Mech. or Man.) as

$$
M_{N(\text { Mech. or Man.) }}=\frac{1}{T} \sum_{j=1}^{T} M_{N_{j}}
$$

Variety is applied to an entire group of ideas and measures how different two ideas are from each other, for each macro functionality. The use of different solutions to satisfy the same function makes two ideas very different. It must be taken into account the four different phases of the genealogy of an idea, similarly to Table 1: 1- Physical principles; 2- Working principles; 3- Embodiment; 4- Detail, to which can be assigned a weight: $W_{l}=10 ; W_{2}=6 ; W_{3}=3 ; W_{4}=1$, as suggested in [7].

It must also be identified the number of varieties $b_{j}$ at each of the four phases. Then the measure of variety can be evaluated for each class (Mech. or Man.), as

$$
M_{V \text { (Mech. or Man.) }}=\sum_{i=1}^{4} f_{i} \cdot \frac{1}{T} \sum_{j=1}^{4} W_{j} b_{j}
$$

Variety reveals a great difference between the two classes: $M_{V}=3.69$ for Management Eng. and $M_{V}=$ 2.12 for Mechanical Eng. This can be associated perhaps to a greater fantasy among students of Management Eng., or better to how students have been engaged in BrainWriting session: conceiving the idea. Students of Mechanical Eng. on the contrary have been invited to decompose the problem and assemble the solution to each functionality. It seems that in the Management Eng. class there has been great freedom in conceiving also strange solutions. On the contrary, Mechanical Eng. students seem to prefer to remain in the comfort zone of more feasible solutions.

Novelty average scores are almost similar, 0.68 for Management Eng. and 0.62 for Mechanical Eng. even there is a certain variability inside each class, as can be seen in Figure 1.

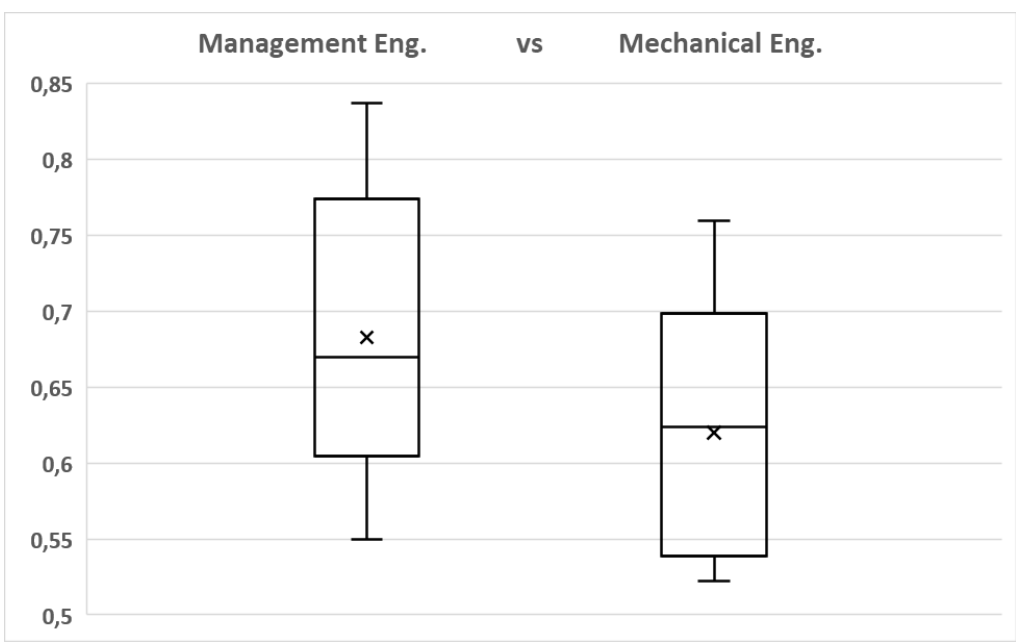

Figure 1. Comparison of novelty score assessed in both classes of Mechanical and Management Engineering students

This means that Mechanical Eng. students, even if they conceived less variability, were able to combine the solutions to each functionality arriving to assemble items that are more novel.

\section{CONCLUSIONS}

The experiment has been performed online, due to the pandemic emergency of COVID-19. Anyway, it has been an interesting experience, that can be repeated, even if a certain lack of empathy among students and teachers reduced exchange of stimuli for creativity. 
The study has been focused on the initial phase of product development and this has shown some differences between the behaviours of the two classes.

Management Engineering students are more inclined to think of original ideas, that can be hardly developed until the detail design phase. They do not consider practical problems that could emerge during product development. Further, it is important to involve management engineering students in product design because they can give a valid aid in the first part of concept design and also, when they will work in firms, they will interact with many stakeholders and may acquire useful information to suggest to development teams.

Mechanical Engineering students are more conscious of all phases of design and seem to be affected by fixation when really, they discard unconsciously ideas that may seems not feasible.

\section{ACKNOWLEDGMENTS}

The authors would like to thank DIMEG at UNICAL for funding such kind of research. They would like to thank all students involved in the experiment. A special thanks to Nino Morabito for his qualified point of view during the assessment.

\section{REFERENCES}

[1] Otto K.N. and Wood K.L. Product Design: Techniques in Reverse Engineering and New Product Development, 2001 (N.J.: Prentice-Hall, Upper Saddle River).

[2] Ulrich K. T. and Eppinger S. D. Product design and development, $5^{\text {th }}$ Ed., 2012, (McGraw-Hill).

[3] Cross N. Engineering design methods, strategies for product design, $3^{\text {rd }}$ Ed., 2000, (John Wiley \&Sons).

[4] De Napoli L., Rizzuti S. and Raco, A. How mood fosters creativity in product design? Experimental evidences on humour impact during a conceptual design session in a master degree class, In: $20^{\text {th }}$ International Conference on Engineering and Product Design Education, E\&PDE 2018, Dyson School of Engineering, Imperial College London, United Kingdom, 6-7 September 2018, pp.242-247.

[5] Rizzuti S. and De Napoli L. Proposal of a Framework Based on Continuous Brainwriting to Expand Mindfulness in Concept Generation. In: Rizzi C., Andrisano A., Leali F., Gherardini F., Pini F., Vergnano A. (eds) Design Tools and Methods in Industrial Engineering - ADM 2019. Lecture Notes in Mechanical Engineering, 2020, pp.352-360, (Springer, Cham).

[6] Shah J. J., Kulkarni S. V. and Vargas-Hernandez N. Evaluation of idea generation methods for conceptual design: effectiveness metrics and design of experiments. Journal of Mechanical Design, 2000, 122(4), 377-385.

[7] Shah J. J. and Vargas-Hernandez N. Metrics for measuring ideation effectiveness. Design Studies, 2003, 24, 111-134.

[8] Linsey J. S., Clauss E. F., Kurtoglu T., Murphy J. T., Wood K. L. and Markman A. B. An experimental study of group idea generation techniques: understanding the roles of idea representation and viewing methods. Journal of Mechanical Design, 2011, 133(3), 031008031008-15.

[9] Dym C. L., Agogino A. M., Eris O., Frey D. D. and Leifer L. J. Engineering design thinking, teaching, and learning. Journal of Engineering Education, 2005, 94(1), 103-120.

[10] https://sketchbook.com/ [Accessed on 2020, 09 March].

[11] Oman S.K., Tumer, I.Y., Wood, K. and Seepersad, C. A comparison of creativity and innovation metrics and sample validation through in-class design projects. Research in Engineering Design, 2013, 24, 65-92.

[12] Runco M.A., Abdulla A.M., Paek S.H., Aljasim F.A. and Alsuwaidi H.N. Which test of divergent thinking is best? Creativity: Theories - Research - Applications, 2016, 3, 4-18.

[13] Amabile T.M. Social psychology of creativity: a consensual assessment technique. Journal of Personality and Social Psychology, 1982, 43 (5), 997-1013.

[14] Torrance E.P. Torrance test of creative thinking: Norms-technical manual, 1974 (Princeton: Personnel Press/Ginn). 